\title{
An Integer Linear Programming model for View Selection on Overlapping Camera Clusters
}

\author{
Massimo Mauro ${ }^{1}$, Hayko Riemenschneider ${ }^{2}$, Alberto Signoroni ${ }^{1}$, Riccardo Leonardi ${ }^{1}$, Luc Van Gool ${ }^{2,3}$ \\ ${ }^{1}$ Department of Information Engineering, University of Brescia, Italia \\ ${ }^{2}$ Computer Vision Laboratory, ETH Zurich, Switzerland \\ ${ }^{3}$ K.U. Leuven, Belgium
}

\begin{abstract}
Multi-View Stereo (MVS) algorithms scale poorly on large image sets, and quickly become unfeasible to run on a single machine with limited memory. Typical solutions to lower the complexity include reducing the redundancy of the image set (view selection), and dividing the image set in groups to be processed independently (view clustering). A novel formulation for view selection is proposed here. We express the problem with an Integer Linear Programming (ILP) model, where cameras are modeled with binary variables, while the linear constraints enforce the completeness of the $3 D$ reconstruction. The solution of the ILP leads to an optimal subset of selected cameras. As a second contribution, we integrate ILP camera selection with a view clustering approach which exploits Leveraged Affinity Propagation $(L A P)$. LAP clustering can efficiently deal with large camera sets. We adapt the original algorithm so that it provides a set of overlapping clusters where the minimum and maximum sizes and the number of overlapping cameras can be specified. Evaluations on four different dataset show our solution provides significant complexity reductions and guarantees near-perfect coverage, making large reconstructions feasible even on a single machine.
\end{abstract}

\section{Introduction}

Scalability is an issue for Multi-View-Stereo (MVS) algorithms $[20,25,7]$ and a limitation for large image-based $3 \mathrm{D}$ reconstructions [1, 4]. A first way to address the problem is to reduce redundancy in the input data. View selection algorithms have the goal of removing repetitive images. However, view selection is not enough when the image set is very large. Since MVS algorithms work by using the whole set of images at once, a further help can come from partitioning the views in clusters to be processed independently and eventually in parallel.

The combination of clustering and selection can lead to three main benefits: 1) reconstruction time on a single ma- chine is reduced due to the smaller dimensions of the clusters; 2) large reconstructions are made possible on single machines with limited memory; 3 ) the processing may be split across multiple machines for further speedups.

In this work ${ }^{1}$, we propose a novel approach for view selection, formulating the problem with an Integer Linear Programming (ILP) model. According to ILP, each camera is represented by a binary variable, which could be selected or not. The linear constraints enforce the shared visibility of points between cameras to guarantee the coverage of the final 3D reconstructions. Solving the ILP model we find a globally optimal set of cameras, avoiding any heuristics or greedy iterative procedures used in other works [26, 9, 8, 6].

As a second contribution, we integrate the ILP selection model with a view clustering solution based on Leveraged Affinity Propagation (LAP). LAP is an extension of Affinity Propagation clustering [5] able to deal with largescale data. We increase the flexibility of the LAP algorithm by handling additional constraints on minimum and maximum cluster size and by admitting overlapping clusters. Cluster overlaps are important for reaching a well-covered reconstruction near cluster boundaries, especially in semistructured camera scenarios [15].

We test our algorithm on different types of image sets: structured, unstructured, streetside and aerial. Experiments show that our method leads to a reduced and well-grouped cameras sets, while guarantees the overall coverage, scalability, and high quality of the $3 \mathrm{D}$ reconstructions.

\subsection{Related Work}

The problem of scalability for large-scale 3D reconstruction has been addressed in a few other works. We briefly discuss the most relevant methods for scaling both Structure-from-Motion (SFM) and Multi-View Stereo (MVS) algorithms.

\footnotetext{
${ }^{1}$ This work was supported by the European Research Council (ERC) under the project VarCity (\#273940) at www.varcity.eu and by the Italian Ministry of Education, University and Research under the PRIN project BHIMM (Built Heritage Information Modeling and Management.)
} 


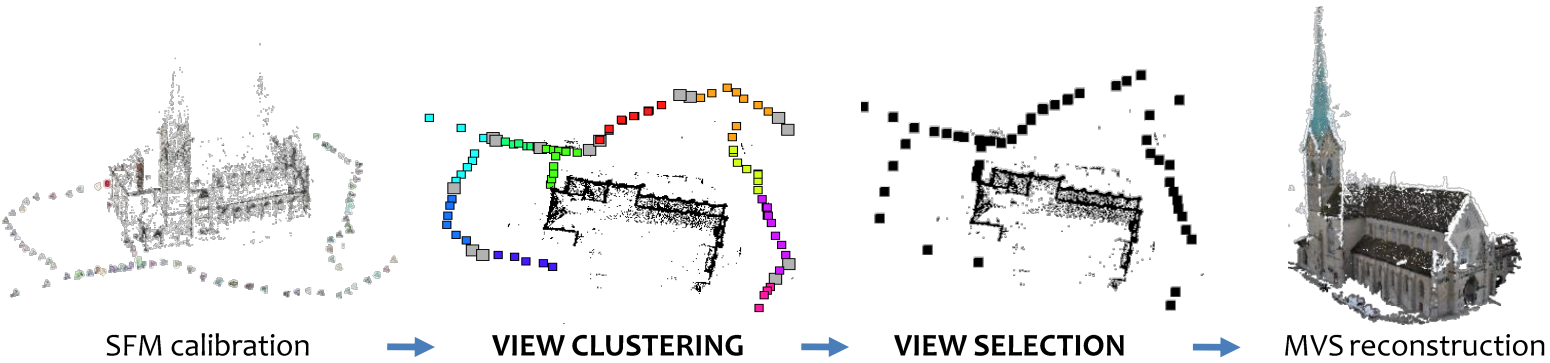

Figure 1. Outline of the view clustering and selection for scalable MVS reconstruction. Different colors indicate different clusters while gray squares are the overlaps. For view selection, black squares show removed cameras.

SFM scalability. Snavely et al. [24] find skeletal sets of images from a given unordered collection which provide a good approximation of the SFM reconstruction using the whole image set. They first estimate reconstruction accuracy between pairs of overlapping images, from which they form a graph and find the skeletal by means of a maximum-leaf t-spanner algorithm. Li et al. [14] find a small subset of iconic images comprising all the important part of the scene. They initially proceed by applying 2D appearance-based constraints to loosely group images, and progressively refining these groups with geometric constraints to select iconic images giving a sparse visual summary of the scene. Crandall et al. [3] introduce a Markov Random Field (MRF) formulation for SFM which finds a coarse initial solution and then improve that solution using bundle adjustment. Their formulation incorporates various sources of information such as noisy geotags or vanishing point estimates. Contrary to these, our work deals with the scalability for MVS and exploits the information about $3 \mathrm{D}$ points and cameras derived from SFM. Hence, the presented approach could be potentially combined with any of the above methods.

MVS scalability. Clustering and selection techniques have been proposed for addressing MVS scalability. In their selection method, Hornung et al. [11] rely on coverage and visibility cues to guarantee a minimum reconstruction quality and then refine the most difficult regions using photoconsistency. Tingdahl et al. [26] reduce the set of initial views relying on depth maps data. Goesele et al. [9] and Gallup et al. [8] select viewpoints relying on simple properties of the input images such as resolution or baseline. Ladikos et al. [13] propose a spectral clustering approach which incorporates scene and camera geometry to build a similarity matrix and then use mean shift to automatically select the number of clusters. Recently, Riemenschneider $e t$ $a l$. [19] provide a partial view selection method which selects parts of an image for the goal of optimizing scene understanding.

Most closely related to our work of view clustering and selection method are the works of Furukawa et al. [6] and Mauro et al. [15]. Furukawa et al. [6] model the problem as an iterative optimization. At first they remove redundant images, then they build a graph representation of remaining cameras and divide them into clusters through normalizedcuts while respecting a constraint on the maximum size of clusters. As a final step, an image addition process creates overlaps between clusters to respect a coverage constraint.

In contrast to them, we place clustering before selection. In this way selection is run on a smaller camera set, and both selection and reconstruction can be parallelized. Since our clustering finds very precise overlaps on cluster borders, selection can be safely parallelized: being careful not to remove overlapping cameras, the creation of holes between clusters can be reliably avoided. As a second difference from Furukawa et al. [6], our ILP selection model is not iterative: it jointly evaluates all cameras and constraints finding an optimal global solution.

Mauro et al. [15] also build a graph based on camera angle similarity and find regular overlapping clusters using dominant sets [18] (DS). View selection is not included in their work. The DS approach requires an iterative procedure: a single run of DS divides the graph in two, a dominant set and a "remaining set". Multi-cluster subdivision is then obtained by iterating on the remaining set. In some cases, this process may isolate some cameras leading to a wrong assignment of cameras to clusters (Figure 2).

Conversely, we adopt Affinity Propagation clustering [5] which considers all data points at once and avoids the above problem. For large image sets, we use Leveraged AP, a version of AP which finds a solution on sampled subset of data and then infers cluster assignment for the whole set.

Hence, the contributions of our paper are the following:

1. a novel optimal formulation of selection with Integer Linear Programming,

2. a novel overlapping cluster assignment exploiting Leveraged Affinity Propagation,

3. the integration of our ILP selection and clustering in a joint system of which we release the code. 


\section{Overlapping view clustering with AP}

The goal of camera clustering is to produce an appropriate number of groups to be processed independently and in parallel. Additionally to the inherent intra-similarity requirements, we want to satisfy the following constraints:

- minimum size constraint: every cluster must be greater than $N_{\min }$ cameras (with $N_{\min } \geq 2$ for making matching possible).

- maximum size constraint: every cluster must be smaller than $N_{\max }$. This constraints allows to run memory expensive dense reconstructions on machines with limited memory capabilities.

- overlap constraint: every cluster must indicate a number $N_{\text {overlap }}$ of overlapping cameras with other clusters. This procedure improves the density of the reconstruction at the "borders" of a cluster.

We divide the presentation of the clustering algorithm in three sub-parts: the point cloud pre-processing, the definition of the camera similarity matrix, our novel multi-cluster solution with constraints.

\subsection{Pre-processing of the SFM point cloud}

We are given a set $C$ of $N_{C}$ cameras and a 3D sparse point cloud $P$ of size $N_{P}$ resulting from Structure-fromMotion (SFM). Considering a camera $C_{i}$, we note as $V_{C_{i}}$ the set of points which are visible from the camera (camera visibility). Dually, considering a point $P_{i}$, we note as $V_{P_{i}}$ the set of cameras looking at the point (point visibility).

Cloud scaling. As a first step, the input point cloud is normalized. The normalization scales the point cloud to have a unit distance $(\bar{R}=1)$ as average distance between its points and their nearest neighbor. This allows the consequent methods to use distances regardless of scale. Also the camera positions are scaled accordingly.

Merging visibility information. As also noted by [6], undetected or unmatched image features may lead to errors in the visibility estimates: adjacent points may have different point visibility sets, leading to imperfect similarity estimates. We thus simplify the structure with a 3D grid voxelization: the new merged points are positioned at the centroids of voxel cells with side dimension $L$, while the point visibilities becomes the union of the point visibilities inside each voxel. Reducing the size of the $P_{S F M}$ cloud lowers the computational effort of the subsequent steps.

\subsection{Similarity matrix}

The $N \times N$ symmetric matrix $S$ of pairwise similarities between cameras takes into account both angle and distance information and is defined as $S=S_{\alpha} \cdot S_{d}$, where $S_{\alpha}$ is the angle matrix and $S_{d}$ is the distance matrix.

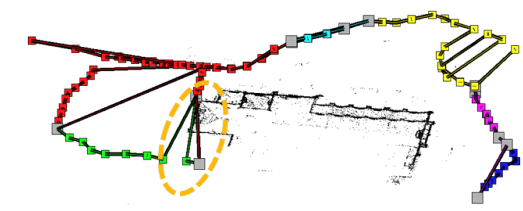

(a) Dominant Sets

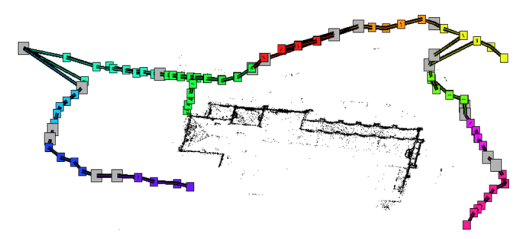

(b) Affinity Propagation

Figure 2. Clustering results with line connections on Fraumunster dataset [15]. Wrong cluster assignments due to the iterative DS clustering are solved with AP.

Angle matrix. Given a pair of cameras $\left(C_{i}, C_{j}\right)$, the angle similarity $s_{\alpha_{i, j}}$ is defined as:

$$
s_{\alpha_{i, j}}=\frac{\sum_{p \in\left(V_{C_{i}} \cap V_{C_{j}}\right)} s_{\alpha_{i j p}}}{\left|V_{C_{i}} \cap V_{C_{j}}\right|}
$$

where $p \in P$ and $s_{\alpha_{i j p}}$ is dependent on the angle $\alpha_{i j p}$ between the viewing directions $\overrightarrow{C_{i}-p}$ and $\overrightarrow{C_{j}-p}$ defined as

$$
\begin{aligned}
s_{\alpha_{i j p}} & =\exp \left(-\frac{\alpha_{i j p}^{2}}{\sigma^{2}}\right) \\
\alpha_{i j p} & =\arccos \frac{\left(C_{i}-p\right)^{T}\left(C_{j}-p\right)}{\left\|C_{i}-p\right\|\left\|C_{j}-p\right\|}
\end{aligned}
$$

There is an angle limit between $30^{\circ}$ and $40^{\circ}$ beyond which the same point is difficult to match among different images [17]. We thus set $\sigma=30^{\circ}$ in our experiments.

Distance matrix. All the values in $S_{\alpha}$ are in the range $[0,1]$. To have the same range in $S_{d}$ we first evaluate the median distance $\bar{d}$, i.e. the median value of the distances between every camera and all the other cameras. Then we define the distance similarity $s_{d_{i, j}}$ as:

$$
s_{d_{i, j}}= \begin{cases}1 & \text { if } i=j \\ \frac{1}{1+\exp \left(-\frac{\left(D\left(C_{i}, C_{j}\right)-\bar{d}\right)}{d}\right)} & \text { otherwise }\end{cases}
$$

where $D$ is the $L_{2}$ norm between the camera centers.

\subsection{Affinity Propagation for overlapping clusters}

Affinity Propagation (AP) considers all given data points at once. As already said, this solves a potential issue of the DS approach [15]. In some situations, such as ordered or semi-ordered camera sets, the iterative process involved by DS may isolate some cameras leading to a wrong assignment of cameras to clusters. An example is in Figure 2. 


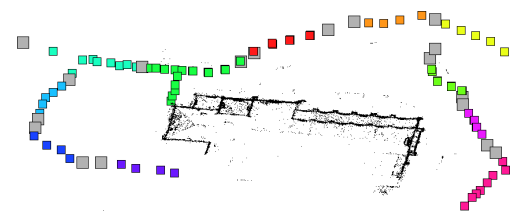

(a) $N_{\text {overlap }}=2$



(b) $N_{\text {overlap }}=4$

Figure 3. Affinity propagation clustering with increasing overlap constraints on Fraumunster dataset. Note the presence of overlaps on both borders of all clusters.

AP takes as input the similarities between data points and simultaneously considers all data as potential exemplars (i.e. cluster centers). Real-valued messages are exchanged between data points until a high-quality set of exemplars and corresponding clusters gradually emerges. Affinity propagation also considers a real $p(k)$ for each data point $k$ so that data points with larger values of $p(k)$ are more likely to be chosen as exemplars. These values are referred to as preferences. The number of identified exemplars (i.e. the number of clusters) is influenced by the values of the input preferences (usually taken as the median of the affinities), but more importantly it also emerges from the message-passing procedure.

Two kinds of message are exchanged between data points, called responsibility and availability. When the algorithm terminates, the responsibility $r(i, k)$, between data point $i$ and exemplar point $k$, reflects how well-suited the data point is as a member of its cluster (how much the point "likes" its exemplar). The availability $a(i, k)$ between exemplar point $k$ and point $i$, reflects how good the candidate exemplar is for the point. We refer to [5] for more details.

Cluster constraints. The original AP algorithm automatically finds the appropriate number of exemplars, and all the cluster form disjoint groups. Instead we need overlapping clusters with pre-determined sizes.

Size constraint. First, too small cluster are merged to nearest clusters - i.e. clusters with the nearest exemplar until the minimum size constraint is satisfied. Second, for too large clusters, we increase preference values by forcing large clusters to be split into two.

Overlap constraint. In the similar vein as [15], we implement a diverse overlapping approach and find a specified number of cluster borders. The first cluster border is selected as the most dissimilar camera to the cluster exemplar, according to $S$. The next borders are iteratively chosen as the least similar cameras to the previous selected ones. A



(a) Speed

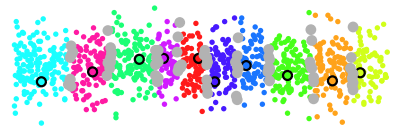

(b) AP Clusters (1000 points)

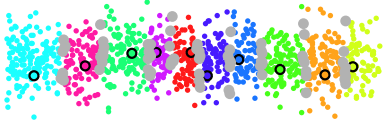

(c) LAP Clusters (1000 points) Figure 4. Speed and cluster comparison between Average Propagation and Leveraged AP.

diverse selection ensures that in semi-structured scenarios both cluster borders are covered by overlaps, see Figure 3.

Leveraged Affinity Propagation. For a large number of cameras, we adopt the so-called Leveraged Affinity Propagation. This is a better option w.r.t. sparse AP, since it is not known a priori if the similarity matrix $S$ is sparse. Leveraged Affinity Propagation samples from the full set of potential similarities and performs several rounds of affinity propagation, iteratively refining the samples. A comparison of AP and LAP methods is done in Figure 4: the speed is compared for randomly generated data of different sizes, while the clustering results are shown for a set of 1000 random points. LAP delivers the same clustering results yet provide a speedup for large datasets.

\section{The ILP model for View Selection}

Integer Linear Programming (ILP) is an optimization model where both the objective function and the constraints are linear, and the variables are integer numbers. The problem of view selection fits perfectly into an ILP model: our variables are the cameras which could be selected or not, reducing ILP to a binary combinatorial problem.

The previous clustering step with precise overlaps allows to run the selection independently on every cluster. As a result the complexity of the ILP optimization problem, which depends on the number of variables, is reduced. We note as $\left\{C_{n}\right\}$ the set of cameras associated with cluster $n$, and $\left\{P_{n}\right\}$ the set of seen points (the union of camera visibilities). Three constraints are specified for view selection:

- coverage constraint: image selection must not create holes or missing parts in the structure;

- size constraint: the resulting number of images in a cluster must satisfy the minimum size constraint $N_{\min }$ specified for clustering; 




Figure 5. Scheme of the needed process for the formulation of the coverage constraint. Different edge widths in the camera subgraph represent the associated similarities. See text for explanation.

- overlap constraint: overlapping cameras in a cluster must be selected, to maintain the reconstruction quality at cluster borders.

All the constraints are explained as linear inequalities in the ILP model. The resulting ILP problem is formalized as:

$\begin{array}{lll}\text { minimize } & \mathbf{c}^{\mathrm{T}} \mathbf{x} & \text { (objective function) } \\ \text { subject to } & A \mathbf{x} \geq \mathbf{b} & \text { (coverage constraint) } \\ & C \mathbf{x} \geq \mathbf{d} & \text { (size constraint) } \\ & E \mathbf{x}=\mathbf{f} & \text { (overlap constraint) }\end{array}$

and $\quad \mathbf{x} \in \mathbf{0}, \mathbf{1}$

Vectors $\mathbf{x}$ and $\mathbf{c}$ are the camera vector and the cost vector respectively, both of length $N_{C_{n}}=\left|\left\{C_{n}\right\}\right|$. The cost vector c is filled with $1 \mathrm{~s}$, so that the total cost is simply the number of selected cameras. However, any other cost attribution can be used, e.g. by attributing different importance according to the estimated saliency [16, 22].

Coverage constraint. Coverage is the most critical among the three constraints. Given the set of cameras in a cluster, one could express the coverage constraint by ensuring that all the $N_{P_{n}}$ points of cluster $n$ are seen by at least $N_{v i s}$ cameras in their point visibility sets (with $N_{v i s} \geq 2$ ). Such a constraint is potentially dangerous, because it implicitly assumes that all cameras in the point visibility set can be matched to each other. This may not be true: especially in semi-structured scenarios where cameras are almost along a line, the angle between cameras at different borders of the same cluster may be too wide for a successful matching. What the coverage constraint then needs to ensure is that all the $N_{P_{n}}$ points of cluster $n$ are seen by at least $N_{\text {vis }}$ matchable cameras in their point visibility sets.

For a linear formulation, we extract for each point $P_{i}$ the subgraph $G_{i}$ of cameras in point visibility set $\left\{V_{P_{i}}\right\}$. Graph $G_{i}$ is edge-weighted, with weights assigned according to values in $S$. From $G_{i}$ we derive an unweighted graph $\tilde{G}_{i}$ by placing a threshold $T_{\text {match }}$ on edge values, such that cameras which are connected in $\tilde{G}_{i}$ can be assumed to be matchable. We then extract all maximal cliques from $\tilde{G}_{i}$ with the Bron-Kerbosch algorithm [2].
The coverage constraint is defined by ensuring that every point in $P_{n}$ is seen by (at least) $N_{v i s}$ cameras in one of its point visibility maximal cliques. Since in a maximal clique every node is connected to all other nodes by definition, retaining $N_{v i s}$ cameras from one of the cliques is the minimum condition to guarantee the coverage. The condition for coverage requires the use of alternative constraints: for every point $P_{i}$ there will be a number of alternative constraints equal to the number of maximal cliques $N_{c l}$ in its camera subgraph $G_{i}$, reflecting the fact that the condition needs to be respected indifferently for only one of the cliques.

The definition of either - or conditions in an ILP model is made possible with the introduction of auxiliary binary variables $y_{1}, \ldots, y_{N_{c l}}$ into the constraint equations.

$$
\begin{gathered}
\sum_{j \in N_{C_{n}}} a_{1 j} x_{j} \geq b_{1}+M_{1}\left(1-y_{1}\right) \\
\ldots \\
\sum_{j \in N_{C_{n}}} a_{N_{c l} j} x_{j} \geq b_{N_{c l}}+M_{N_{c l}}\left(1-y_{N_{c l}}\right) \\
\sum_{k \in\left[1 \ldots N_{c l}\right]} y_{k} \geq 1 \quad y_{k} \in 0,1
\end{gathered}
$$

where $a_{k, j}=1$ only when camera $C_{j}$ belongs to the point visibility maximal clique of the considered point (otherwise $a_{k, j}=0$ ), and $b_{k}=N_{v i s}$. By specifying $M_{k}=-N_{v i s}$ and with $y_{k}=0$, the constraint on clique $k$ is weakened as the right-hand side of the inequality renders the condition always true. Conversely, an $y_{k}=1$ "activates" the constraint. We then impose that at least one of alternative constraints is "active" to guarantee the coverage for the point. A graphical representation of the needed steps for the coverage constraint is in Figure 5.

Size constraint. Size constraint is set such that the sum of $x_{i} \in \mathrm{x}$ is greater then the minimum cluster size. Hence, $C$ is filled with 1 s and $d=N_{\text {min }}$.

Overlap constraint. This condition forces the overlapping cameras to be selected. Matrix $E$ reduces to a vector where $e_{i, j}=1$ for overlapping cameras (otherwise $\left.e_{i, j}=0\right)$ and $f=N_{\text {overlaps }}$. 




(a) CMVS

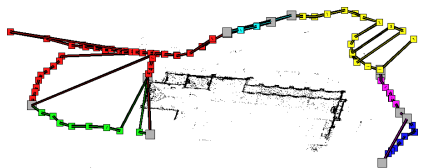

(b) DS

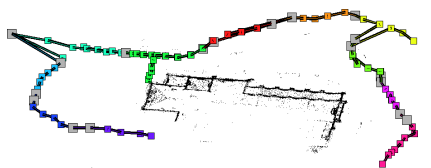

(c) AP

Figure 6. Affinity propagation clustering compared to CMVS [6] and DS [15] on Fraumunster dataset.

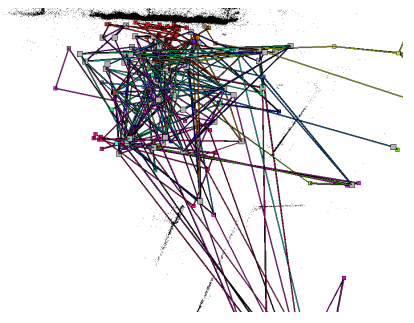

(a) CMVS

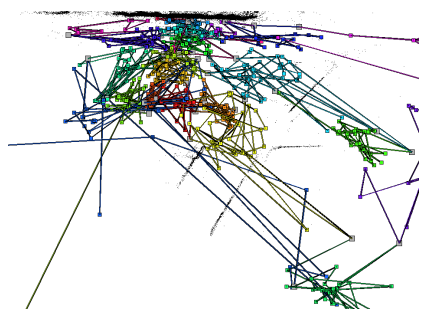

(b) DS

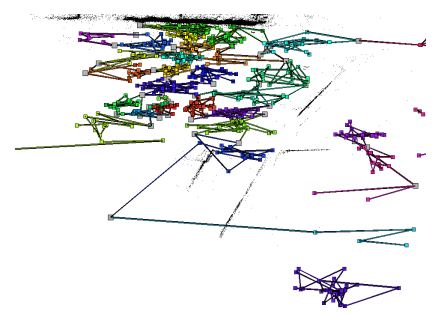

(c) AP

Figure 7. Affinity propagation clustering compared to CMVS [6] and DS [15] on Notredame dataset.

\section{Experimental Evaluation}

In this section, we experimentally validate the clustering and selection methods. For clustering, we highlight the regularity of clusters and overlaps compared to other two recent methods: CMVS [6] and DS [15]. As to selection we compare the full CMVS tool based on heuristics for selection and evaluate the coverage of the obtained 3D models and the runtime gains to a reconstruction run on the full set. We test on four different datasets corresponding to four different scenarios: Fraumunster [15] (structured landmark), Yotta [21] (streetside), Notre Dame (unstructured landmark) [23], and Wundschuh [10] (aerial).

Parameters Settings. There a few parameters to define in our method. The parameter that most affect the the final results is the voxel dimension $L$ (Section 2.1): increasing this value, the selection algorithm has more room for removal since the camera subgraph associated to every point is bigger. After some preliminary experiments, we set a $L=15 \bar{R}$ as we found to guarantee good coverage performances for all datasets. We set $N_{\max }=40, N_{\min }=3$ and $N_{\text {overlaps }}=2$ as clustering parameters. As regards selection, we use $T_{\text {match }}=0.7$ as threshold for the derivation of the edge-unweighted graph in Section 3 , and $N_{v i s}=2$ as the minimum number of cameras seeing every point.

\subsection{Clustering}

Clustering results are shown for Fraumunster and NotreDame in Figures 3, 6, 7. In Figure 3 clustering results are shown using two different $N_{\text {overlaps }}$ values, highlighting the precise positions of cluster overlaps on borders. In Figure 6 and 7 cameras in each cluster are connected with a line to better compare the regularity of the clusters in the three different methods. In general, our clusters and overlaps always show a better cluster configuration w.r.t. CMVS as we model angle and distance and not just pure shared visibility. DS improves upon CMVS, but still some wrong assignments remain due to the iterative problem. Cluster compactness is beneficial during selection, since every cluster has little influence on the others and thus selection can be run independently on each of them. Moreover, the precision of overlaps avoids any problem of incomplete reconstructions between clusters.

\subsection{Selection}

To quantitatively evaluate image selection, we would require a ground truth, which is not readily available for these experiments as there is no best minimal set of images with an optimal point cloud. In this work, for every dataset we reconstruct a 3D point model with the Patch-based MultiView Stereo (PMVS) method [7] using the complete set of images. This most complete point cloud reconstruction serves as reference.

We define coverage as a metric for evaluation. Given a ground truth point cloud $\mathcal{G}$, and a point cloud $\mathcal{P}$, coverage is computed as follows: for every point $g_{i}$ in $G$, we evaluate the distance $d_{G P}$ to the nearest point in $\mathcal{P}$. The point $g_{i}$ is "covered" if such distance is below a given threshold $\overline{d_{G P}}$. The coverage metric is given by the percentage of covered points in $G$. We set $\overline{d_{G P}}=4 \bar{R}$.

Quantitative results are shown in Table 1. We obtain almost complete coverage with respect to the PMVS computed on the full set. Compared to CMVS, we generally improve on coverage performances. At the same time, we achieve important speed-up factors, overall up to $8.5 \mathrm{x}$ achieved on the NotreDame and up to $84 \%$ faster than CMVS for Yotta dataset. Gains are obtained without parallelization, thus much greater speedup are easily achievable by splitting the reconstruction on multiple machines. 


\begin{tabular}{|c|c|c|c|c|}
\hline $\begin{array}{c}\text { Dataset } \\
\text { Type }\end{array}$ & $\begin{array}{c}\text { Fraumunster } \\
\text { structured }\end{array}$ & $\begin{array}{c}\text { NotreDame } \\
\text { unstructured }\end{array}$ & $\begin{array}{c}\text { Yotta } \\
\text { streetside }\end{array}$ & $\begin{array}{c}\text { Wundschuh } \\
\text { aerial }\end{array}$ \\
\hline \# images & 98 & 706 & 380 & 307 \\
\hline CMVS \# clusters & 5 & 12 & 13 & 5 \\
Our \# clusters & 11 & 36 & 15 & 23 \\
\hline CMVS \# selected & 55 & 279 & 356 & 143 \\
Our \# selected & 49 & 272 & 199 & 131 \\
\hline CMVS Coverage & $97.9 \%$ & $96.5 \%$ & $93.8 \%$ & $96.6 \%$ \\
Our Coverage & $98.7 \%$ & $97.2 \%$ & $93.8 \%$ & $96.8 \%$ \\
\hline CMVS Speedup & $2.7 x$ & $8.3 x$ & $3.2 x$ & $4.0 \mathrm{x}$ \\
Our Speedup & $\mathbf{3 . 0 x}$ & $\mathbf{8 . 5 x}$ & $\mathbf{5 . 9 x}$ & $\mathbf{4 . 9 x}$ \\
Relative Speedup & $\mathbf{1 1 1 \%}$ & $\mathbf{1 0 3 \%}$ & $\mathbf{1 8 4 \%}$ & $\mathbf{1 2 3 \%}$ \\
\hline
\end{tabular}

Table 1. Quantitative results on four different datasets.

The ILP optimizations are modeled and solved with the LP-solve package[12]. The runtime of the entire system is between $0.5 \mathrm{~min}$ (Fraumunster, smallest) and $3 \mathrm{~min}$ (NotreDame, largest dataset) which is negligible w.r.t. the full PMVS reconstruction with 0.75 hour (Fraumunster) and 8 hours (NotreDame), respectively.

For a qualitative evaluation of the selection method, Figure 8 shows cluster subdivisions and removed cameras after selection for all datasets, highlighting the regularity of removed cameras. The good coverage properties of our method are also confirmed by a visual analysis of 3D reconstructions: in Figure 9 the 3D models obtained by using the full image set and our subset are nearly identical.

\section{Conclusions}

In this work we presented an approach for joint camera clustering and selection, in order to improve Multi-ViewStereo (MVS) scalability.

Two novel methods are introduced. First, we exploit Leveraged Affinity Propagation for clustering, extending the original algorithm to manage cluster and overlaps constraints. The resulting clusters and diverse overlaps are regular and well-defined, comparing favorably with other stateof-the-art methods [6, 15]. Second, we introduce an Integer Linear Programming formulation for view selection. The ILP model treats cameras as binary variables and jointly handles all necessary constraints finding a global solution.

The two methods are combined and it is shown that the final set of clustered and selected cameras ensures a nearlyperfect coverage of the 3D scene and leads to large speedup factors (up to 8x, without parallel processing) compared to MVS reconstructions computed on the full image set.

As future work, we plan to reason about regions of interest within the images rather than always considering the full images during selection.

\section{References}

[1] S. Agarwala, Y. Furukawaa, N. Snavely, I. Simon, B. Curless, S. M. Seitz, and R. Szeliski. Building rome in a day. Commmunications of ACM, 54(10), 2011. 1
[2] C. Bron and J. Kerbosch. Algorithm 457: finding all cliques of an undirected graph. Communications of the ACM, 16(9):575-577, 1973. 5

[3] D. Crandall, A. Owens, N. Snavely, and D. Huttenlocher. Discrete-continuous optimization for large-scale structure from motion. In $C V P R$, pages 3001-3008. IEEE, 2011. 2

[4] J.-M. Frahm, P. Georgel, D. Gallup, T. Johnson, R. Raguram, C. Wu, Y.-H. Jen, E. Dunn, B. Clipp, S. Lazebnik, and M. Pollefeys. Building rome on a cloudless day. In ECCV. 2010. 1

[5] B. J. Frey and D. Dueck. Clustering by passing messages between data points. science, 315(5814):972-976, 2007. 1, 2, 4

[6] Y. Furukawa, B. Curless, S. M. Seitz, and R. Szeliski. Towards internet-scale multi-view stereo. In $C V P R, 2010.1,2$, 3, 6, 7

[7] Y. Furukawa and J. Ponce. Accurate, dense, and robust multiview stereopsis. In $C V P R, 2007.1,6$

[8] D. Gallup, J.-M. Frahm, P. Mordohai, and M. Pollefeys. Variable baseline/resolution stereo. In CVPR, 2008. 1, 2

[9] M. Goesele, N. Snavely, B. Curless, H. Hoppe, and S. Seitz. Multi-view stereo for community photo collections. In CVPR, 2007. 1, 2

[10] C. Hoppe, A. Wendel, S. Zollmann, K. Pirker, A. Irschara, H. Bischof, and S. Kluckner. Photogrammetric Camera Network Design for Micro Aerial Vehicles. In $C V W W, 2012$. 6

[11] A. Hornung, B. Zeng, and L. Kobbelt. Image selection for improved multi-view stereo. In $C V P R, 2008.2$

[12] http://lpsolve.sourceforge.net/. 7

[13] A. Ladikos, S. Ilic, and N. Navab. Spectral camera clustering. In $I C C V, 2009.2$

[14] X. Li, C. Wu, C. Zach, S. Lazebnik, and J.-M. Frahm. Modeling and recognition of landmark image collections using iconic scene graphs. In ECCV, pages 427-440. Springer, 2008. 2

[15] M. Mauro, H. Riemenschneider, L. Van Gool, and R. Leonardi. Overlapping camera clustering through dominant sets for scalable $3 \mathrm{~d}$ reconstruction. In $B M V C, 2013$. 1, $2,3,4,6,7$

[16] M. Mauro, H. Riemenschneider, L. Van Gool, A. Signoroni, and R. Leonardi. A unified framework for content-aware view selection and planning through view importance. In BMVC, 2014. 5

[17] K. Mikolajczyk, T. Tuytelaars, C. Schmid, A. Zisserman, J. Matas, F. Schaffalitzky, T. Kadir, and L. Van Gool. A 


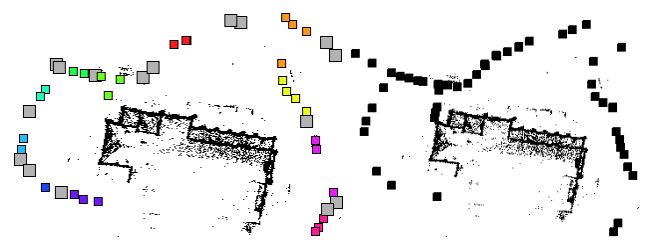

(a) Fraumunster



(b) Yotta

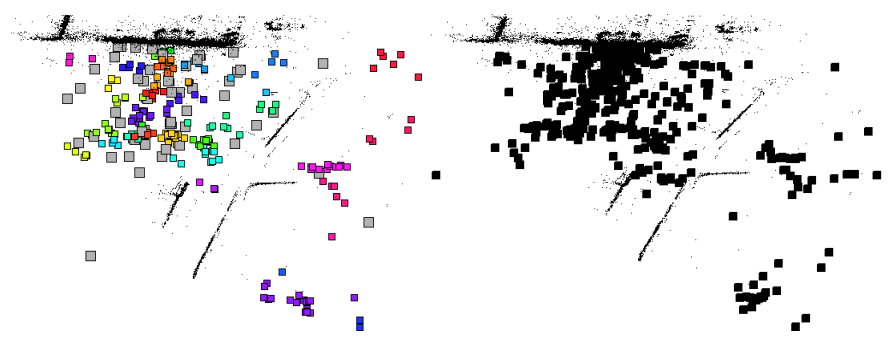

(c) Notre Dame

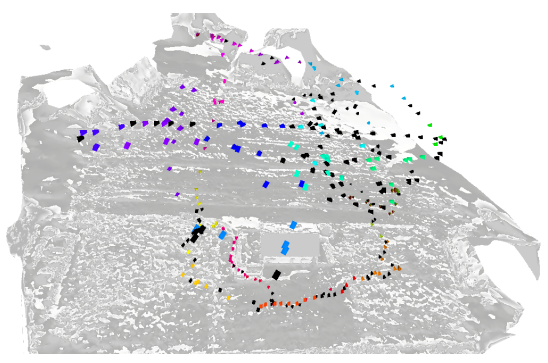

(d) Wundschuh

Figure 8 . Selection results on the four datasets. Removed cameras in black.
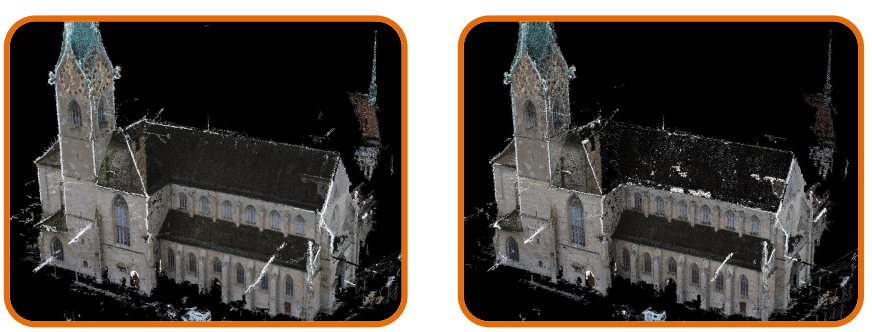

(a) Fraumunster
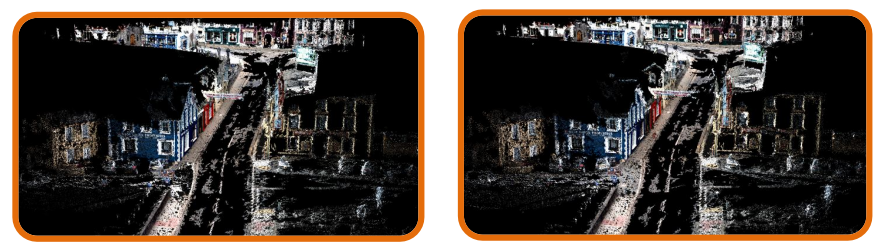

(c) Yotta

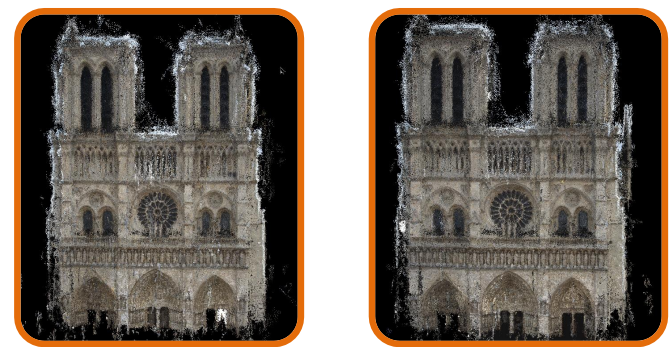

(b) NotreDame
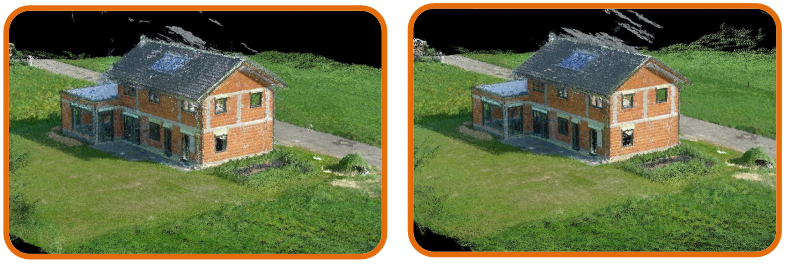

(d) Wundshcuh

Figure 9. Comparison of reconstruction results obtained on the four datasets when using the full set (left) and the selected set (right).

comparison of affine region detectors. IJCV $, 65(1-2): 43-72$, 2005. 3

[18] M. Pavan and M. Pelillo. Dominant sets and pairwise clustering. Pattern Analysis and Machine Intelligence, IEEE Transactions on, 29(1):167-172, 2007. 2

[19] H. Riemenschneider, A. Bodis-Szomoru, J. Weissenberg, and L. Van Gool. Learning Where To Classify In Multi-View Semantic Segmentation. In ECCV, 2014. 2

[20] S. Seitz, B. Curless, J. Diebel, D. Scharstein, and R. Szeliski. A comparison and evaluation of multi-view stereo reconstruction algorithms. In CVPR, 2006. 1

[21] S. Sengupta, P. Sturgess, L. Ladicky, and P. Torr. Automatic Dense Visual Semantic Mapping from Street-Level Imagery. In IROS, 2012. 6

[22] E. Shtrom, G. Leifman, and A. Tal. Saliency detection in large point sets. In $I C C V, 2013.5$
[23] N. Snavely, S. Seitz, and R. Szeliski. Modeling the World from Internet Photo Collections. IJCV, 80(2):189-210, 2007. 6

[24] N. Snavely, S. M. Seitz, and R. Szeliski. Skeletal graphs for efficient structure from motion. In $C V P R$, volume 1, page 2, 2008. 2

[25] C. Strecha, W. von Hansen, L. Van Gool, P. Fua, and U. Thoennessen. On benchmarking camera calibration and multi-view stereo for high resolution imagery. In $C V P R$, 2008. 1

[26] D. Tingdahl and L. Van Gool. A public system for image based $3 \mathrm{~d}$ model generation. In Computer $\mathrm{Vi}$ sion/Computer Graphics Collaboration Techniques, pages 262-273. Springer, 2011. 1, 2 